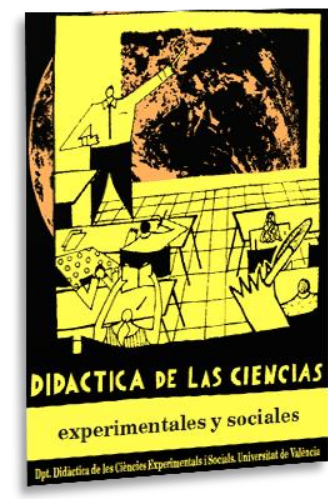

\title{
Clima del aula en la Educación Científica
}

\section{Classroom Environment in Science Education}

DOI: $10.7203 / D C E S .35 .11410$

\author{
Irene Espadero \\ Universitat de València, iesber@alumni.uv.es
}

Amparo Vilches

Universitat de València, amparo.vilches@uv.es

ORCID iD: https://orcid.org/0000-0001-5308-2714

RESUMEN: El clima del aula es un aspecto esencial que impregna todo el proceso de enseñanza y aprendizaje, desde el papel que el profesorado debe jugar en el aula, en sus diferentes dimensiones, hasta las relaciones que mantiene con su alumnado y las que ayuda a generar entre ellos. A lo largo del trabajo se analizan diversos factores que influyen en el clima del aula, estudiando su evolución desde que en los años 80 se comenzara a hablar del tema en la didáctica de las ciencias. Se estudia si en la actualidad se le presta la debida atención para, en caso negativo, realizar propuestas que puedan mejorar la enseñanza-aprendizaje de las ciencias a partir de la intervención en este ámbito.

Palabras Clave: clima del aula, aspectos axiológicos, clima del centro, alfabetización científica de la ciudadanía, trabajo colaborativo

ABSTRACT: The classroom environment is an essential aspect present in the whole teaching-learning process, from the role of the teacher in the classroom, in its different dimensions, to the relationships held with the students and among students. Along this paper some factors that affect classroom environment will be analyzed, studying its evolution since they were first mentioned in the $80 \mathrm{~s}$ in science education. The aim of this article is to analyze if classroom environment is currently properly considered, in order to include some intervention proposals to improve the science teaching-learning process if necessary.

KEYWORDS: classroom environment, axiological aspects, school environment, scientific literacy for citizenship, collaborative work

Fecha de recepción: enero de 2018

Fecha de aceptación: julio de 2018 


\section{INTRODUCCIÓN}

El estudio del clima de aula, en particular en la enseñanza de las ciencias, tiene sus orígenes en las primeras consideraciones sobre escuelas eficaces en los años 80. La investigación en torno a las Effective School Research surgió como un movimiento que se oponía a la idea impulsada por Coleman y otros (1966) acerca de la escasa influencia que las variables escolares ejercían en el rendimiento de los estudiantes (Báez, 1994). Este enfoque se centró en analizar los procesos psicosociales y las interacciones que se desarrollan en el ámbito de la institución escolar. Supuso dejar de considerar que el "problema" está en el alumnado y plantear el relevante papel que juega la institución escolar y los métodos de uso de sus recursos disponibles (Rivas, 1986).

Sammons, Hillman y Mortimore (1995), en su revisión sobre la investigación en torno a escuelas eficaces, destacan el clima de aula como un aspecto esencial. Así, defienden que el espíritu de una escuela está también determinado por el clima en el que los estudiantes trabajan, caracterizado por una atmósfera ordenada y un ambiente de trabajo atractivo. Así mismo, reflejan la importancia de una visión y metas comunes, las altas expectativas y los refuerzos positivos, una monitorización del progreso de los estudiantes y que los alumnos y alumnas se sientan responsables de su propio aprendizaje.

Desde entonces, el clima de aula ha sido señalado en la investigación en Didáctica de las Ciencias como un tema fundamental y de vital importancia a la hora de favorecer el aprendizaje del alumnado. Welch (1985) incluía el clima escolar y el clima de aula entre las siete líneas de investigación que aparecían en la literatura como más prometedoras por su posible incidencia positiva en el aprendizaje de las ciencias (Gil et al., 1991). El clima del aula constituía ya un tema relevante al que se le prestaba atención en el Handbook of Research on Science Teaching and Learning (Gabel 1994), en un artículo publicado por Fraser (1994). Más recientemente, es considerada una de las once áreas significativas del campo de la educación científica en el 2nd International Handbook of Science Education (Fraser, Tobin y McRobbie, 2012), dedicando en la Parte 9 ocho capítulos a distintos aspectos de los ambientes de aprendizaje. En el primero de los capítulos, Classroom Learning Environments: Retrospect, Context and Prospect (Fraser, 2012) se describe la situación actual referente a los ambientes de aprendizaje en torno a qué son, cómo evolucionan, etc., mostrando instrumentos específicos para evaluar las percepciones de los ambientes de aprendizaje en el aula y en el centro. En el capítulo siguiente, Teacher-Students relationships in the classroom (Wubbels y Brekelmans, 2012), se analizan las relaciones entre docentes y estudiantes señalando que la forma en que los docentes interactúan con el alumnado no solo predice sus logros, sino que también se relaciona con factores como la satisfacción laboral del profesorado o el síndrome Burnout.

\section{Planteamiento del Problema}

Si bien existe un consenso entre los especialistas de que el clima de aula es un aspecto fundamental a tener en cuenta en cualquier ámbito educativo, cabe destacar su importancia en la educación científica, dado el rechazo al que se enfrenta a menudo por parte del alumnado (Solbes y Vilches, 1997; Solbes, 2011; Vázquez y Manassero, 2011). El Informe Rocard (2007), promovido por la Comisión Europea debido al descenso del interés por estudios científicos por parte de los jóvenes, ofreció una serie de recomendaciones para ser tenidas en cuenta por los países europeos, señalando que el origen de este descenso del interés está, en buena medida, en la forma en que la ciencia es enseñada, lo que supone prestar atención a la formación del profesorado, que constituye la piedra angular de cualquier renovación de la educación científica. En el informe se señala la necesidad de sustituir las estrategias de transmisión recepción por otras que orienten el aprendizaje, y la formación del profesorado, como una tarea de indagación o investigación, que favorezca la participación de los estudiantes en la re-construcción de los conocimientos, algo que concita hoy un 
consenso general entre los expertos (Vilches y Gil Pérez, 2007). A este respecto, por tanto, el clima de aula adquiere una gran importancia, pues debe contribuir a la inmersión en la cultura científica y a favorecer el interés hacia la misma (Gil-Pérez et al., 2005), superando visiones reduccionistas de la propia formación del profesorado.

La importancia concedida al clima del aula nos llevó a investigar si, en la actualidad, se le presta atención adecuada en la enseñanza de las ciencias. Más concretamente, nos propusimos dar respuesta a una serie de preguntas en relación con el clima del aula: ¿Es un tema que preocupa y del que se ocupa el profesorado de ciencias o de física y química en particular? ¿Qué percepciones tienen los estudiantes sobre la importancia del clima del aula? ¿Hasta qué punto son tratados estos aspectos en la investigación y en los materiales didácticos? ¿Se le presta atención en la formación del profesorado?

El objetivo fundamental de este estudio es analizar la situación para, en su caso, preparar en una siguiente fase materiales y herramientas de intervención en la formación del profesorado para que contribuyan a una mayor implicación de los docentes en la atención al clima del aula, por su importancia para un mejor aprendizaje del alumnado y aumentar el interés hacia la ciencia y su estudio.

\section{HIPÓTESIS QUE ORIENTA EL ESTUDIO Y SU FUNDAMENTACIÓN}

Numerosas investigaciones convergentes han venido mostrando una escasa atención en general en la enseñanza de las ciencias a los aspectos axiológicos del aprendizaje, en particular a las interacciones CTSA (Solbes y Vilches, 1997 y 2004; Gil et al., 2005; Vazquez y Manassero, 2011), así como a la necesaria alfabetización científica de la ciudadanía, preparada para participar en la toma de decisiones frente a los graves problemas que afectan a la humanidad (Marco, 2000; Hodson, 2003 y 2011; Acevedo, Vázquez y Manassero, 2003; Gil et al., 2005; Vilches y Gil, 2009). Dichos estudios, entre otros, nos llevaron a pensar que la educación científica no está prestando suficiente atención al clima del aula, lo que constituye la hipótesis fundamental del estudio que presentamos. Ello conlleva una serie de consecuencias que permitirán su contrastación, a través del análisis, entre otros, de la atención dedicada al clima de aula en la investigación en didáctica de las ciencias o por el propio profesorado.

Como ha puesto de manifiesto la investigación, la inmersión en una cultura científica constituye una forma excelente de favorecer la educación científica. Esta propuesta, que supone aproximar el aprendizaje de las ciencias a una investigación, ha sido fundamentada por numerosos autores y aparece como fruto esencial de la investigación en didáctica de las ciencias, reflejado en diversos Handbooks (Gabel, 1994; Fraser y Tobin, 1998; Perales y Cañal, 2000; Abell y Lederman, 2007; Fraser, Tobin y McRobbie, 2012). La necesidad de sustituir las estrategias de transmisiónrecepción por otras que orienten el aprendizaje como una tarea de indagación o investigación, que favorezcan la participación de los estudiantes en la (re)construcción de los conocimientos, concita un consenso amplio entre los expertos. Y en particular se llama la atención sobre aspectos esenciales en la construcción de conocimientos científicos que, a menudo, no son suficientemente tenidos en cuenta en la enseñanza de las ciencias, refiriéndose tanto a aspectos conceptuales como metodológicos, a los problemas de contextualización del trabajo científico (relaciones CTS, toma de decisiones...) y a los componentes afectivos (interés por la tarea, actitudes, clima del aula y del centro, etc.). Entre estos, y como una dimensión esencial vinculada a los aspectos axiológicos, podemos señalar que la atención en particular a la Sostenibilidad, aunque ha ido creciendo en los últimos años y se ha incorporado en el currículum de los diferentes niveles y en la formación del profesorado, es todavía insuficiente, teniendo en cuenta el agravamiento de la situación y la necesidad de la adopción urgente de medidas para la construcción de un presente y futuro sostenibles (Vilches y Gil Pérez, 2009). 
Por lo que se refiere a la formación del profesorado, precisamente, uno de los resultados más relevantes de la Effective School Research (Rivas, 1986) fue que el funcionamiento de las "escuelas eficaces" estaba caracterizado por las altas expectativas que el profesorado de dichos centros poseía y transmitía a su alumnado, así como por el seguimiento y apoyo constante a su trabajo. Es preciso por tanto determinar estrategias formativas que pueden resultar adecuadas para contribuir a un clima adecuado para el aprendizaje, mediante el que los educadores podamos contribuir a la doble tarea de estimular el interés por la cultura científica del conjunto de la ciudadanía y a su vez preparar adecuadamente a un número suficiente de candidatos y candidatas para proseguir estudios científico-tecnológicos superiores. Porque, tal como ha mostrado la investigación (Vilches y Gil Pérez, 2007), no basta con que los docentes modifiquemos nuestra enseñanza ofreciendo una visión más rica y atractiva, es decir, más ajustada a la realidad, de la actividad científica. Hay que tener en cuenta que muchos estudiantes llegan con prejuicios muy enraizados contra los estudios científicos y por tanto, sin algo que ponga en cuestión estos prejuicios, desde el principio, nuestros esfuerzos innovadores pueden fracasar. Es conveniente, por todo ello, abordar la problemática del clima del aula y generar expectativas positivas.

Los resultados de muchos años de investigación en torno a las escuelas eficaces (Rivas, 1986) permiten referirse a las siguientes características de las mismas: un conjunto de objetivos básicos, bien definidos, alcanzables y compartidos por todo el profesorado, para conseguir una acción convergente y de refuerzo mutuo, de forma que se logre un mayor aprovechamiento del tiempo escolar; una estructuración adecuada de las actividades escolares, en la que se encuentran incluidas las cuestiones de dosificación, ritmo y secuencias docentes, así como un nivel de dificultad apropiado de las tareas de aprendizaje; el control de ayuda o acompañamiento docente, que implica la supervisión de la realización de la tarea, la estimulación a través de preguntas pertinentes y la proporción de retroalimentación de manera continua; un proceso de diagnóstico, evaluación y retroacción vinculado a los objetivos y que permita conocer la medida en que han sido alcanzados; una continua atención en sus manifestaciones cognitivas, de información, de motivación y refuerzo, en forma de aceptación de las ideas de los alumnos, etc.; el tiempo escolar de aprendizaje, que requiere una activa implicación del alumnado en las tareas, que estas estén vinculadas a los objetivos, y que experimente una notoria proporción de éxitos; unas altas expectativas educativas de logro de los docentes respecto del alumnado, de sí mismos y de la propia institución escolar; un ambiente escolar ordenado, de autodisciplina compartida y respeto mutuo, etc.

Más recientemente, la investigación ha planteado estrategias concretas que contribuyen a un buen clima de aula y a mejorar el aprendizaje y el interés del alumnado, qué factores influyen en este aprendizaje y cómo enfocarlos. En estos estudios (Gil et al., 2005; Vilches, 2007) se plantea la importancia de establecer una serie de compromisos profesorado-alumnado los primeros días de clase, de forma que se llegue a una serie de acuerdos que todos consensuen y sobre los que se pueda trabajar, prestando una mayor atención a los aspectos más creativos y relevantes de la actividad científica (interacciones CTSA, importancia de los temas a tratar, realización de experiencias sencillas, etc.). Un factor esencial es el de la participación del alumnado, de forma que se fomente el carácter colectivo de la ciencia y por tanto de la educación científica, trabajando en equipos. Por último, también se destaca la importancia del apoyo permanente al trabajo de los estudiantes, alentándoles en sus tareas, de forma que sean capaces de perseverar en la superación de las dificultades, considerando la evaluación como un instrumento fundamental de aprendizaje, que juega un importante papel para la consecución de estos compromisos.

Un aspecto con gran influencia sobre el clima del aula, ampliamente estudiado e investigado, es el del aprendizaje cooperativo o colaborativo. Se trata de un mecanismo de construcción conjunta de significado (Ibáñez y Gómez, 2005; León et al., 2011), que ofrece múltiples ventajas, como la obtención de un aprendizaje más profundo, una mayor expresión y participación del alumnado y una mayor autonomía y autorregulación del propio proceso de aprendizaje. Resulta esencial el papel que juega el trabajo cooperativo en asignaturas científicas, aproximando a la forma en que trabaja la 
ciencia, ofreciendo una visión más coherente de la misma (Fernández et al., 2002; Gómez e Insausti, 2004) y promoviendo la participación del alumnado en las tareas científicas (Gil et al., 2005). No obstante, a pesar de las ventajas de esta herramienta metodológica, esencial por su contribución a un clima de aula adecuado para un aprendizaje significativo, diferentes estudios muestran que su uso no se ha generalizado (Vilches y Gil Pérez, 2011).

\section{DISEÑOS EXPERIMENTALES}

Para poner a prueba la hipótesis, centrada en la idea de la escasa atención prestada, en general, por la educación científica al clima del aula, se diseñaron una serie de instrumentos; un diseño múltiple que trata de encontrar resultados convergentes con la utilización de diferentes herramientas.

\subsection{Análisis de revistas de investigación}

Se pretende una primera aproximación a la atención de la investigación en Educación en Ciencias al clima de aula, analizando los artículos de los años 2011 a 2017 de una selección de revistas de referencia en el ámbito, cuatro españolas y una internacional: Enseñanza de las Ciencias, Revista Eureka sobre Enseñanza y Divulgación de las Ciencias, Revista Electrónica de Enseñanza de las Ciencias, Revista de Educación y International Journal of Science Education.

\subsection{Cuestionario para estudiantes}

Este diseño, consistente en preguntas abiertas (Cuadro 1) dirigidas a estudiantes de Secundaria que ya fue validado en investigaciones precedentes (Gil Pérez et al., 2005; Vilches, 2007), estudia las opiniones que tiene el alumnado de Educación Secundaria y Bachillerato acerca de su proceso de aprendizaje de las ciencias a lo largo de su trayectoria académica, analizando si aparecen aspectos relacionados con el clima de aula y la importancia que le conceden.

CUADRo 1. Cuestionario para estudiantes, sobre el análisis de la enseñanza recibida

Muchos alumnos y alumnas rechazáis los estudios científicos o, al menos, no los encontráis interesantes. Y en parte tenéis razón. Esto tiene causas diversas que conviene analizar y sobre las que es necesario actuar, para evitar que el mundo realmente apasionante de la ciencia (que lo es de verdad) provoque indiferencia y rechazo. En esa situación los docentes hemos de asumir nuestra propia responsabilidad y modificar todo aquello que pueda estar perjudicando una educación científica atractiva y satisfactoria.

1. ¿Qué aspectos de la educación que habéis recibido hasta el momento en las asignaturas científicas encontráis criticables y preferiríais que no continuaran llevándose a cabo?

2. ¿Qué cosas os han gustado o parecido de interés?

3. ¿Qué habéis echado en falta en esa enseñanza o a qué os gustaría que se le diera más importancia?

Con esta herramienta, y teniendo en cuenta estudios precedentes, esperamos que se refirieran a distintos aspectos del proceso de enseñanza y aprendizaje, algunos directamente vinculados con el clima de aula (como las relaciones entre el alumnado y docentes o entre el mismo alumnado, la participación de los estudiantes, etc.) y otros relacionados, ya que tanto la metodología seguida por 
el profesorado como algunos aspectos procedimentales y otros axiológicos influyen de manera directa en el clima de aula, como hemos señalado anteriormente.

\subsection{Entrevista para el profesorado}

Tiene como objetivo analizar la atención prestada por el profesorado de ciencias al clima de aula. Fue validado por expertos del campo de la investigación en didáctica de las ciencias, analizando el grado de coherencia entre los objetivos y los enunciados de los ítems. De acuerdo con la hipótesis planteada, se espera obtener unos resultados que muestren una escasa consideración del clima de aula como factor clave en el aprendizaje del alumnado.

Se diseñó un formato de entrevista (Cuadros 2 y 3) en la que, en una primera parte, sin introducir el tema principal a tratar para evitar sesgos en las respuestas, se plantea una pregunta abierta acerca de cómo favorecer el aprendizaje del alumnado (Cuadro 2). Con ello se quiere poner de manifiesto si el profesorado considera el clima de aula como uno de los factores principales a tener en cuenta a la hora de mejorar el proceso de aprendizaje de las ciencias. En la segunda parte de la entrevista, se introduce ya directamente el tema del clima de aula como un ámbito fundamental e influyente en el aprendizaje del alumnado, instando al docente a dar su opinión mediante la formulación de diferentes preguntas (Cuadro 3).

CUADRO 2. Entrevista previa para profesorado sobre la atención al clima del aula

Con el fin de contribuir a mejorar la Enseñanza de las Ciencias y en particular de la Física y la Química, queremos plantearle la siguiente cuestión:

1. ¿Cómo piensa que se podría favorecer el aprendizaje del alumnado en las clases? ¿Qué aspectos deberíamos tener en cuenta?

CUADRO 3. Cuestionario para estudiantes, sobre el análisis de la enseñanza recibida

Como sabemos, muchos de nuestros alumnos y alumnas llegan con prejuicios a las clases de ciencias, fruto de sus experiencias previas y del clima social, en torno a las dificultades del aprendizaje de las ciencias, que se traducen en actitudes de desinterés, cuando no de rechazo. Si queremos generar una actitud positiva, sin la cual resulta casi imposible un aprendizaje significativo, es necesario contribuir a un clima de aula que favorezca su implicación en el proceso de aprendizaje. El estudio del clima del aula y del centro ha sido abordado, a lo largo de las últimas décadas, por numerosas investigaciones en diferentes campos de la educación, y muy en particular de la educación científica, con numerosas aportaciones, en las que se muestra la gran influencia que dicho clima tiene en el aprendizaje de los estudiantes y en las actitudes hacia la ciencia. Nos gustaría, en ese sentido, conocer su opinión acerca de la importancia del clima del aula y qué propuestas o sugerencias lleva adelante en el aula para conseguir contribuir a un clima adecuado para el aprendizaje de las ciencias.

1. ¿Qué importancia tiene para usted el clima del aula en las clases de ciencias?

2. En su opinión ¿Qué factores piensa que influyen en el clima del aula? 
3. ¿Cómo los orienta para conseguir un clima de aula que contribuya a aumentar el interés de los estudiantes y a mejorar el aprendizaje?

4. ¿Con qué obstáculos o dificultades se enfrenta?

5. ¿Cómo piensa que se podría contribuir a su superación?

\section{Algunos Resultados}

A continuación, mostraremos resultados de la aplicación de algunos de los diseños llevados a cabo hasta el momento en la investigación.

\subsection{Resultados del análisis de las revistas}

Se analizaron artículos publicados, en el período 2011 a 2017, en 5 revistas seleccionadas entre las más utilizadas en el ámbito, localizando aquellos que tengan como tema principal el que nos ocupa (ver Tabla 1). En un segundo nivel de análisis, se han incluido los artículos centrados en temas axiológicos relacionados con el clima de aula, aunque este no aparezca explícitamente. En la tabla I, se muestra el número de artículos analizados de cada revista.

TABLA 1. Artículos analizados en el periodo 2011-2017

\begin{tabular}{cc}
\hline Revista & Número publicaciones (2011-2017) \\
\hline $\begin{array}{c}\text { Enseñanza de las Ciencias (EC) } \\
\text { Revista Eureka }\end{array}$ & 262 \\
\hline $\begin{array}{c}\text { sobre Enseñanza y Divulgación de las Ciencias } \\
\text { (REurEDC) }\end{array}$ & 326 \\
\hline Revista de Educación (RE) & 401 \\
\hline $\begin{array}{c}\text { Revista Electrónica de Enseñanza de las Ciencias } \\
\text { (REEC) }\end{array}$ & 181 \\
\hline $\begin{array}{c}\text { International Journal of Science Education (IJSE) } \\
\text { TOTAL }\end{array}$ & 829 \\
\hline
\end{tabular}

Las siguientes tablas ( 2 a 4) recogen los artículos encontrados que pueden englobarse en el primer nivel de análisis (los centrados en el clima de aula) o en el segundo (relacionados con el clima del aula aunque no estén centrados en él). 
TABLA 2. Artículos que tratan el clima del aula encontrados en las revistas analizadas en el periodo 20112017 (primer nivel de análisis)

\begin{tabular}{|c|c|c|}
\hline Revista & Año & Artículo \\
\hline $\mathrm{EC}$ & 2012 & $\begin{array}{l}\text { Perseguer, C. (2012). Identificación de los elementos de gestión del aula mediante } \\
\text { la investigación-acción. Enseñanza de las Ciencias 30(3), 257-279. }\end{array}$ \\
\hline REEC & 2016 & $\begin{array}{l}\text { Tiago, D. e Buso, C. (2016). Dinâmicas de grupo em aulas de biología: uma } \\
\text { proposta motivacional para a aprendizagem. Revista Electrónica de Enseñanza de } \\
\text { las Ciencias 15(1), 147-166. }\end{array}$ \\
\hline \multirow[t]{4}{*}{ IJSE } & 2016 & $\begin{array}{l}\text { Silva, D. and McNeill, K. (2016). Secondary science students' beliefs about class } \\
\text { discussions: a case study comparing and contrasting academic tracks. International } \\
\text { Journal of Science Education 38(12), 2047-2068. }\end{array}$ \\
\hline & 2016 & $\begin{array}{l}\text { Fung, D. and Wai-mei, L. (2016). Individual to collaborative: guided group work } \\
\text { and the role of teachers in junior secondary science classrooms. International } \\
\text { Journal of Science Education 38(7), 1057-1076. }\end{array}$ \\
\hline & 2012 & $\begin{array}{l}\text { Chen, S. F. et al. (2012). A cross-grade comparison to examine the context effect } \\
\text { on the relationships among family resources, school climate, learning participation, } \\
\text { science attitude, and science achievement based on TIMMSS } 2003 \text { in Taiwan. } \\
\text { International Journal of Science Education 34(14), 2089-2106. }\end{array}$ \\
\hline & 2011 & $\begin{array}{l}\text { Raved, L. and Assaraf, O.B.Z. (2011). Attitudes towards science learning among } \\
10^{\mathrm{t}} \text {-grade students: a qualitative look. International Journal of Science Education } \\
33(9), 1219-1243 \text {. }\end{array}$ \\
\hline
\end{tabular}

TABLA 3. Artículos relacionados con la atención al clima del aula encontrados en las revistas analizadas en el periodo 2011-2017 (segundo nivel de análisis)

\begin{tabular}{ccl}
\hline Revista & Año & \multicolumn{1}{c}{ Artículo } \\
\hline EC & 2017 & $\begin{array}{l}\text { García Ferrandis, I., Vilches, A. y García Ferrandis, X. (2017). Estrategias } \\
\text { didácticas innovadoras en la enseñanza de las ciencias. Estudio de caso: los } \\
\text { maestros del Patronato de Educación Rural de Valencia (1958-1985), Enseñanza } \\
\text { de las Ciencias, 35 (2), 109-126. }\end{array}$ \\
\cline { 2 - 3 } & 2014 & $\begin{array}{l}\text { Mellado, V. et al., (2014). Las emociones en la enseñanza de las ciencias. } \\
\text { Enseñanza de las Ciencias 32(3), 11-36. }\end{array}$
\end{tabular}

2013 Hugo, D., Adúriz-Bravo, A. y Sanmartí, N. (2013). Estilos de trabajo emocional del futuro profesorado de ciencias durante el prácticum. Enseñanza de las Ciencias 31(1), 151-168.

2012 Sanz, I., Llorens, J. y Llorens J.A. (2012). La caracterización del ambiente de aprendizaje en un laboratorio de Química General mediante métodos de investigación social. Enseñanza de las Ciencias 30(1), 5-22.

2011 Rodrigues, A., Mattos C. (2011). Contexto, negociación y actividad en una clase de física. Enseñanza de las ciencias 29(2), 263-274. 


\begin{tabular}{|c|c|c|}
\hline \multirow[t]{5}{*}{ REurEDC } & 2017 & $\begin{array}{l}\text { Dávila-Acedo, M.A. (2017). Las emociones y sus causas en el aprendizaje de } \\
\text { Física y Química, en el alumnado de Educación Secundaria Revista Eureka sobre } \\
\text { Enseñanza y Divulgación de las Ciencias 14(3), 570-586. }\end{array}$ \\
\hline & 2016 & $\begin{array}{l}\text { Arandia, E., Zuza, K. y Guisasola, G. (2016). Actitudes y motivaciones de los } \\
\text { estudiantes de ciencias en Bachillerato y Universidad hacia el aprendizaje de la } \\
\text { física. Revista Eureka sobre Enseñanza y Divulgación de las Ciencias 13(3), 558- } \\
\text { 573. }\end{array}$ \\
\hline & 2015 & $\begin{array}{l}\text { Garmendia, M. y Guisasola, J. (2015). Alfabetización científica en contextos } \\
\text { escolares: El Proyecto Zientzia Live! Revista Eureka sobre Enseñanza y } \\
\text { Divulgación de las Ciencias 12(2), 294-310. }\end{array}$ \\
\hline & 2013 & $\begin{array}{l}\text { Costillo, E. et al., (2013). Las emociones sobre la enseñanza-aprendizaje de las } \\
\text { ciencias y las matemáticas de futuros profesores de Secundaria. Revista Eureka } \\
\text { sobre Enseñanza y Divulgación de las Ciencias } 10,514-532 \text {. }\end{array}$ \\
\hline & 2012 & $\begin{array}{l}\text { De Longhi, A. L. et al., (2012). La interacción comunicativa en clases de ciencias } \\
\text { naturales. Un análisis didáctico a través de circuitos discursivos. Revista Eureka } \\
\text { sobre Enseñanza y Divulgación de las Ciencias } 9(2), 178-195 \text {. }\end{array}$ \\
\hline \multirow[t]{5}{*}{$\mathrm{RE}$} & 2017 & $\begin{array}{l}\text { Rodríguez, D., Armengol, C. y Meneses, J. (2017). La adquisición de las } \\
\text { competencias profesionales a través de las prácticas curriculares de la formación } \\
\text { inicial de maestros. Revista de Educación 376, 229-251. }\end{array}$ \\
\hline & 2012 & $\begin{array}{l}\text { Ceballos, E. M. et al., (2012). La voz del alumnado en el conflicto escolar. Revista } \\
\text { de Educación 359, 554-579. }\end{array}$ \\
\hline & 2012 & $\begin{array}{l}\text { Mata, P. y Ballesteros B. (2012). Diversidad cultural, eficacia escolar y mejora de } \\
\text { la escuela: encuentros y desencuentros. Revista de Educación } 358,17-37 \text {. }\end{array}$ \\
\hline & 2012 & $\begin{array}{l}\text { Moliner, O. et al., (2012). Las medidas específicas de atención a la diversidad en } \\
\text { la Educación Secundaria Obligatoria (ESO) desde las percepciones de los agentes } \\
\text { implicados, Revista de Educación } 358,197-217 \text {. }\end{array}$ \\
\hline & 2011 & $\begin{array}{l}\text { Martínez, M., Esteban, F. y Burraxais, M.R. (2011). Escuela, profesorado y } \\
\text { valores. Revista de Educación extraordinario 2011. Educación, valores y } \\
\text { democracia, 95-113. }\end{array}$ \\
\hline REEC & 2012 & $\begin{array}{l}\text { Faria, C. and Chagas, I. (2012). School-visit to a science centre: student } \\
\text { interaction with exhibits and the relevance of teachers' behavior. Revista } \\
\text { Electrónica de Enseñanza de las Ciencias 11(3), 582-594. }\end{array}$ \\
\hline \multirow[t]{2}{*}{ IJSE } & 2017 & $\begin{array}{l}\text { Bathgate, M., \& Schunn, C. (2017). The psychological characteristics of } \\
\text { experiences that influence science motivation and content } \\
\text { knowledge. International Journal of Science Education 39(17), 2402-2432. }\end{array}$ \\
\hline & 2017 & $\begin{array}{l}\text { Melo, L., Cañada, F., and Mellado, V. (2017). Exploring the emotions in } \\
\text { Pedagogical Content Knowledge about the electric field. International Journal of } \\
\text { Science Education 39(8) } 1-20 \text {. }\end{array}$ \\
\hline
\end{tabular}


2017 Wang, C. L., and Liou, P. Y. (2017). Students' motivational beliefs in science learning, school motivational contexts, and science achievement in Taiwan. International Journal of Science Education 39(7), 898-917.

2017 Klemm, J., and Neuhaus, B. J. (2017). The role of involvement and emotional well-being for preschool children's scientific observation competency in biology. International Journal of Science Education 39(7), 863-876.

2016 Navarro, M. et al., (2016). Attitudes toward science: measurement and psychometric properties of the Test of Science-Related Attitudes for its use in Spanish-speaking classrooms. International Journal of Science Education 38(9), 1459-1482.

2016 Eliasson, N., Sorensen, H. and Göran, K. (2016). Teacher-student interaction in contemporary science classrooms: is participation still a question of gender? International Journal of Science Education 38(10), 1655-1672

2016 Lu, Y., Chen, H., Hong, Z. and Yore, L. (2016) Students' awareness of science teachers' leadership, attitudes toward science, and positive thinking. International Journal of Science Education 38(13), 2174-2196.

2015 King, D. et al., (2015). Emotionally intense science activities. International Journal of Science Education 37(12), 1886-1914.

2013 Logan, M.R. and Skamp, K.R. (2013). The impact of teachers and their science teaching on students 'science interest': a four-year study. International Journal of Science Education 35(17), 2879-2904.

2013 Yung, B.H. et al., (2013). Teachers' and students' conceptions of good science teaching. International Journal of Science Education 35(14), 2435-2461.

2013 Day, S.P. and Bryce, T.G. (2013). The benefits of cooperative learning to socioscientific discussion in secondary school science. International Journal of Science Education 35(9), 1533-1560.

2013 Kim, M. and Tan, H.T. (2013). A collaborative problem-solving process through environmental field studies. International Journal of Science Education 35(3), 357-387.

Como puede observarse, existen pocos trabajos centrados o con referencias al Clima del Aula, lo que nos indica una tendencia de escasa atención a la problemática en la muestra de revistas analizadas. La siguiente tabla (Tabla 4) muestra el porcentaje de artículos que abordan o están relacionados con el clima del aula con respecto al total de artículos consultados.

TABLA 4. Artículos de las revistas de investigación que hacen referencia al Clima del Aula

\begin{tabular}{ccc}
\hline $\begin{array}{c}\text { Análisis de artículos con referencia al Clima } \\
\text { de Aula (N=1999) }\end{array}$ & $\begin{array}{c}\text { Número de } \\
\text { contribuciones }\end{array}$ & $\begin{array}{c}\text { \% respecto del total de } \\
\text { artículos consultados }(\delta)\end{array}$ \\
\hline Primer nivel & 6 & $0.3 \%(0.1)$ \\
\hline Segundo nivel & 28 & $1.4 \%(0.3)$ \\
\hline Total & 34 & $1.43 \%(0.3)$
\end{tabular}


Por otro lado, en la muestra seleccionada y en otras revistas del ámbito, se han encontrado artículos que, a pesar de no tratar explícitamente el clima de aula, incluyen o se refieren a ejemplos de buenas prácticas o de atención a otros temas axiológicos muy relacionados y mutuamente influyentes, como el aprovechamiento de la educación no formal, lo relacionado con las emociones (Melo, Cañada y Mellado, 2017; Retana Alvarado et al., 2018) la motivación y actitudes de los estudiantes (Arandia, Zuza y Guisasola, 2016), relaciones CTSA o Educación para la Sostenibilidad (Vilches y Gil Pérez, 2009), que esperamos poder tener en cuenta en la segunda fase de esta investigación, en la orientación de las propuestas que se pretenden fundamentar.

\subsection{Resultados del cuestionario aplicado a estudiantes}

Fue utilizado con una muestra de 124 estudiantes de $3^{\circ}$ de ESO, $1^{\circ}$ y $2^{\circ}$ de Bachiller de dos centros de Valencia, uno público y otro concertado. Se llevó a cabo un primer análisis cuantitativo, en el que se contabilizan y agrupan las respuestas de los estudiantes a cada una de las preguntas formuladas, en base a las siguientes categorías: aspectos metodológicos del aprendizaje, aspectos procedimentales y aspectos axiológicos/clima de aula. A pesar de la separación que aquí se plantea, todos los aspectos están muy relacionados y se influyen mutuamente. Así, los metodológicos resultan imprescindibles para configurar un buen clima de aula y por tanto no pueden considerarse independiente de este. De la misma manera, los aspectos procedimentales, además de tratarse de aspectos metodológicos, configuran una pieza fundamental en esa creación de un clima de aula adecuado.

En las siguientes tablas 5 a 7, puede observarse el número de estudiantes que han hecho alusión a los diferentes aspectos en cada una de las preguntas. La suma de los porcentajes para cada curso excede el $100 \%$ ya que se puede hacer alusión a diferentes aspectos dentro de la misma respuesta a una pregunta.

TABLA 5. Aspectos mencionados por los estudiantes en sus respuestas a la primera pregunta del cuestionario: ¿Qué aspectos de la educación que habéis recibido hasta el momento en las asignaturas científicas encontráis criticables y preferiríais que no continuaran llevándose a cabo?

\begin{tabular}{cccc}
\hline Aspectos & $3^{\circ}$ ESO N=41 & Bachillerato N=83 & Total de estudiantes N=124 \\
\hline Metodológicos & $20(48.8 \%)$ & $53(63.9 \%)$ & $73(58.9 \%)$ \\
\hline Procedimentales & $8(19.5 \%)$ & $15(18.1 \%)$ & $23(18.5 \%)$ \\
\hline Axiológicos. Clima de aula & $9(22 \%)$ & $19(22.9 \%)$ & $28(22.6 \%)$ \\
\hline Otros & $1(2.4 \%)$ & $11(13.3 \%)$ & $12(9.7 \%)$ \\
\hline NS/NC & $14(34.2 \%)$ & $10(12 \%)$ & $24(19.4 \%)$ \\
\hline
\end{tabular}

TABLA 6. Aspectos mencionados por los estudiantes en sus respuestas a la segunda pregunta del cuestionario: ¿Qué cosas os han gustado o parecido de interés?

\begin{tabular}{cccc}
\hline Aspectos & $3^{\circ}$ ESO N=41 & Bachillerato N=83 & Total de estudiantes N=124 \\
\hline Metodológicos & $23(56 \%)$ & $34(41 \%)$ & $57(46 \%)$ \\
Procedimentales & $15(36.6 \%)$ & $27(32.5 \%)$ & $42(33.9 \%)$ \\
\hline Axiológicos. Clima de aula & $4(9.8 \%)$ & $37(44.6 \%)$ & $41(33.1 \%)$ \\
\hline Otros & 0 & $1(1.2 \%)$ & $1(0.8 \%)$ \\
\hline NS/NC & $5(12.19 \%)$ & $9(10.8 \%)$ & $14(11.3 \%)$ \\
\hline
\end{tabular}


TABLA 7. Aspectos mencionados por los estudiantes en sus respuestas a la tercera pregunta del cuestionario: ¿Qué habéis echado en falta en esa enseñanza o a qué os gustaría que se le diera más importancia?

\begin{tabular}{cccc}
\hline Aspectos & $3^{\circ}$ ESO N=41 & Bachillerato N=83 & Total de estudiantes N=124 \\
\hline Metodológicos & $4(9.8 \%)$ & $21(25.3 \%)$ & $25(20.2 \%)$ \\
\hline Procedimentales & $20(48.8 \%)$ & $50(60.2 \%)$ & $70(56.5 \%)$ \\
\hline Axiológicos. Clima de aula & $8(19.5 \%)$ & $22(26.5 \%)$ & $30(24.2 \%)$ \\
\hline Otros & $1(2.4 \%)$ & $5(6 \%)$ & $6(4.8 \%)$ \\
\hline NS/NC & $12(29.3 \%)$ & $3(3.6 \%)$ & $15(12.1 \%)$
\end{tabular}

Aunque el alumnado de ESO presenta respuestas más escuetas y generales al de Bachillerato, que como era de esperar sus aportaciones son más ricas, los resultados son convergentes. No solo el número de aspectos relacionados con el clima de aula es elevado, lo que indica la importancia dada por los estudiantes a este aspecto fundamental del proceso de enseñanza aprendizaje, sino también los metodológicos y procedimentales con influencia directa sobre él. Así, critican una metodología excesivamente teórica, con escasa participación y limitada a escuchar, copiar y resolver ejercicios, en la que indican que se ven obligados a memorizar cosas innecesarias que muchas veces hacen sin entender. Evidentemente, todos estos aspectos metodológicos no pueden obviarse si quiere propiciarse un buen clima de aula. De igual forma, la realización de experiencias, que los estudiantes califican de muy escasa, juega un papel clave en la motivación de los estudiantes. Por último, muchos aspectos propios del clima de aula aparecen entre las respuestas de los estudiantes: el papel del docente (si ayuda, si atiende a los alumnos, si desmotiva), el escaso trabajo en grupo, o la ausencia de una introducción ante una nueva asignatura o tema son algunos ejemplos. En el caso de los estudiantes de segundo de bachillerato el profesorado juega un papel más importante, pues los consultados valoran su implicación, la motivación que despierta en los estudiantes, su trato cercano y su ánimo hacia ellos. Aparecen otras ideas relacionadas con aspectos axiológicos, como realizar proyectos científicos o investigaciones, exposiciones, etc., que sin duda contribuyen a crear un clima de aula propicio para un aprendizaje significativo y el interés hacia el estudio de la ciencia.

Estos resultados, más de diez años después, son convergentes con los obtenidos en el periodo de 2002 a 2004 en otros estudios e investigaciones, dirigidos al planteamiento de actividades para el principio de curso (Gil et al., 2005; Vilches, 2007), al aplicar el mismo cuestionario los primeros días de clase a muestras de 98 estudiantes de $3^{\circ}$ y $4^{\circ} \mathrm{de}$ ESO y del Bachillerato de centros públicos de la ciudad de Valencia, así como a 30 estudiantes del último curso de la Facultad de Física, y a 87 futuros docentes de física y química que estaban realizando el CAP (Curso de Aptitud Pedagógica) en la Universitat de València. La situación no parece haber cambiado mucho en los años transcurridos.

\subsection{Resultados de las entrevistas al profesorado de ciencias}

Se realizó la entrevista a seis docentes en activo de centros públicos y concertados de dos comunidades autónomas diferentes, separando la entrevista previa de la específica sobre el clima de aula, tal como se indicó anteriormente, para evitar sesgos en las respuestas.

Respecto a la primera parte, los docentes se refieren al tipo de alumnado, su motivación o interés. También aparecen aspectos metodológicos, como la importancia de llevar a cabo clases prácticas y experimentales, las dificultades que supone la herramienta matemática o el número de alumnos por aula como factor determinante. Sin embargo, a excepción de uno de los docentes que menciona las interacciones profesor-alumno, ninguno señala explícitamente aspectos relativos al clima del aula como un factor favorecedor del aprendizaje. Este patrón de respuestas va en la línea 
de nuestra hipótesis, pues el hecho de que un docente no lo plantee es síntoma de que no lo trabaja o no le da suficiente importancia.

En la segunda parte de la entrevista, se introduce al docente directamente en el tema de la investigación, pidiéndole su opinión en torno a diferentes cuestiones. Los entrevistados coinciden al ser preguntados en la importancia que tiene el clima del aula para el aprendizaje del alumnado y un buen aprovechamiento de las sesiones. Aparecen algunas aportaciones a cerca de lo que entienden por Clima del aula con ideas relativas a "ser amigo de los alumnos", o "no ser un sargento". Cabe destacar igualmente que, aunque todos los docentes han considerado el clima de aula esencial para el aprendizaje de los alumnos, ninguno de ellos explícitamente lo había mencionado en la pregunta previa, además en sus respuestas parece entreverse que el clima del aula no es algo que se pueda y deba trabajar sino que "es como es" y si es un mal clima se trabaja peor y poco se puede hacer, ya que se da a entender en sus respuestas al segundo y tercer ítem que la responsabilidad es de los estudiantes exclusivamente: "Si entras en un grupo que la mayoría son estudiosos, pues generalmente [...] resulta más fácil dar clase, porque esos arrastran a los demás. Si entras en un grupo donde hay unos cuantos alumnos que te boicotean la clase, entonces conseguir un buen clima es muy difícil [...]".

Al avanzar en la reflexión, en el ítem 3, se encuentran ideas relativas al clima del aula como la importancia de las relaciones de respeto sin autoritarismos para crear un buen clima, así como la importancia de introducir aspectos de su vida cotidiana que les ayuden a conectar con la asignatura. Dos docentes destacan también la importancia del trabajo colaborativo y otro de los entrevistados señala aspectos fundamentales en la configuración de un clima de aula adecuado, como son la presencia de unas normas o pautas que los alumnos han aceptado y la necesidad de trabajar en esto desde el principio.

Entre los principales obstáculos para la consecución de un buen clima de aula, referidos a las respuestas al ítem 4, destacan de nuevo el elevado número de alumnos por aula, la falta de tiempo y la presión por cumplir con un programa (sobre todo en bachillerato), la falta de motivación o de interés por parte del alumnado, y las dificultades idiomáticas que pueden sufrir los inmigrantes (y sus profesores). Observamos de nuevo la tendencia de los entrevistados al señalar los problemas en los estudiantes y no en el propio papel del docente.

Por lo que se refiere al último ítem, que pretende conocer sus ideas acerca de cómo superar las dificultades del clima de aula para que contribuya a un mejor aprendizaje, en general los docentes implicados insisten en que la responsabilidad sobre el clima del aula es de los estudiantes. Entre las principales soluciones, destacan de nuevo la importancia de disminuir la ratio, o la posibilidad de hacer desdobles; así como un mayor uso de los laboratorios. Dos de los docentes coinciden en la necesidad de separar antes a los alumnos según sus aptitudes o intereses, de forma que puedan elegir lo que consideren más apropiado. Uno añade un matiz digno de mención: "Sin estos alumnos en las aulas ordinarias de la ESO, se contribuiría muy positivamente al funcionamiento de los grupos".

\section{UNAS PRIMERAS CONCLUSIONES Y PERSPECTIVAS}

En esta investigación nos planteamos estudiar si en la actualidad se está otorgando la atención adecuada al Clima del Aula en la enseñanza de las ciencias, aspecto fundamental y prometedora línea de investigación señalada por distintos Handbooks en las últimas décadas. El primero de los diseños llevados a cabo, consistente en el análisis de los artículos publicados durante los cinco últimos años en una selección de revistas, ha aportado unos resultados en la línea de la hipótesis de la escasa atención al clima del aula en la educación científica, pues de un total de 1999 artículos consultados, únicamente 34 trataban de manera directa el clima del aula o sobre todo algún tema relacionado. El análisis tanto cuantitativo como cualitativo de las respuestas del alumnado implicado ha reflejado sin embargo el peso de algunos aspectos metodológicos y procedimentales 
que, sin duda alguna, tienen una gran influencia en la configuración de un adecuado clima de aula. Todo esto sostiene la idea de lo importante y relevante que resulta para el alumnado esta cuestión en su proceso de aprendizaje. Por último, las entrevistas realizadas a docentes de ciencias en activo muestran la escasa atención prestada al Clima del Aula, atribuyendo los problemas, en general, a aspectos ajenos a ellos sin reconocer su parte de responsabilidad en la consecución de un clima adecuado para el aprendizaje.

Para terminar, queremos señalar que este trabajo no pretende solo analizar la situación, sino que se tiene previsto preparar, poner en marcha y evaluar materiales para la formación del profesorado de forma que el proceso de enseñanza y aprendizaje pueda verse mejorado y favorecido por la atención adecuada un aspecto tan esencial como es el Clima del Aula.

\section{Referencias}

Abell, S.K. y Lederman, N.G. (Eds.) (2007). Handbook of Rresearch on Science Education. Mahwah, NJ: Lawrence Erlbaum.

Acevedo, J.A., Vázquez, A. y Manassero, M.A. (2003). Papel de la educación CTS en una alfabetización científica y tecnológica para todas las personas, REEC: Revista Electrónica de Enseñanza de las Ciencias, 2(2), 80-111.

Arandia, E., Zuza, K. y Guisasola, G. (2016). Actitudes y motivaciones de los estudiantes de ciencias en Bachillerato y Universidad hacia el aprendizaje de la física. Revista Eureka sobre Enseñanza y Divulgación de las Ciencias, 13(3), 558-573.

Báez, B.F. (1994) El movimiento de escuelas eficaces; implicaciones para la innovación educativa Revista Iberoamericana de Educación, 4, 93-116.

Bathgate, M. y Schunn, C. (2017). The psychological characteristics of experiences that influence science motivation and content knowledge. International Journal of Science Education, 39(17), 2402-2432.

Ceballos, E. M., (2012). La voz del alumnado en el conflicto escolar. Revista de Educación, 359, 554-579.

Chen, S. F., Lin, C.Y., Wang, J.R., Lin, S.W. y Kai, H.L. (2012). A cross-grade comparison to examine the context effect on the relationships among family resources, school climate, learning participation, science attitude, and science achievement based on TIMMSS 2003 in Taiwan. International Journal of Science Education, 34(14), 2089-2106.

Coleman, J. S., Campbell, E., Hobson, C., Mcpartland, J., Mood, A., Weinfeld, F. y York, R. (1966). Equality of educational opportunity. Washington: U.S. Government Printing Office.

Costillo, E., Borrachero, B., Brígido, M. y Mellado, V. (2013). Las emociones sobre la enseñanzaaprendizaje de las ciencias y las matemáticas de futuros profesores de Secundaria. Revista Eureka sobre Enseñanza y Divulgación de las Ciencias, 10, 514-532.

Dávila-Acedo, M.A. (2017). Las emociones y sus causas en el aprendizaje de Física y Química, en el alumnado de Educación Secundaria. Revista Eureka sobre Enseñanza y Divulgación de las Ciencias, 14(3), 570-586.

Day, S.P. y Bryce, T.G. (2013). The benefits of cooperative learning to socio-scientific discussion in secondary school science. International Journal of Science Education, 35(9), 1533-1560.

De Longhi, A. L., Ferreyra, A., Peme, C., Bermúdez, G.M.A., Quse, L., Martínez, S., Iturralde, C. y Campaner, G. (2012). La interacción comunicativa en clases de ciencias naturales. Un análisis didáctico a través de circuitos discursivos. Revista Eureka sobre Enseñanza y Divulgación de las Ciencias, 9(2), 178-195.

Eliasson, N., Sorensen, H. y Göran, K. (2016). Teacher-student interaction in contemporary science classrooms: is participation still a question of gender? International Journal of Science Education, 38(10), 1655-1672 
Faria, C. y Chagas, I. (2012). School-visit to a science centre: student interaction with exhibits and the relevance of teachers' behavior. Revista Electrónica de Enseñanza de las Ciencias, 11(3), 582-594.

Fernández, I., Gil-Pérez, D., Carrascosa, J., Cachapuz, J. y Praia, J. (2002). Visiones deformadas de la ciencia transmitidas por la enseñanza. Enseñanza de las Ciencias, 20(3), 477-488.

Fraser, B. (1994). Research on classroom and school climate. En D. Gabel (Ed.), Handbook of Research on Science Teaching and Learning. Nueva York: McMillan Pub Co.

Fraser B. (2012). Classroom Learning Environments: Retrospect, Context and Prospect, In Fraser, Tobin, McRobbie (Eds.) Second International Handbook of Science Education, Dordrecht: Springer, 1191-1239.

Fraser, B. J. y Tobin K. G. (Eds.) (1998). International Handbook of Science Education London: Kluber Academic Publishers.

Fraser, B., Tobin, K. y Mcrobbie, C. J. (Eds.) (2012). Second international handbook of science education (Vol. 24). Dordrecht: Springer.

Fung, D. y Wai-Mei, L. (2016). Individual to collaborative: guided group work and the role of teachers in junior secondary science classrooms. International Journal of Science Education, 38(7), 1057-1076.

Gabel, D. (1994). Handbook of Research on Science Teaching and Learning. New York: McMillan.

García Ferrandis, I., Vilches, A. y García Ferrandis, X. (2017). Estrategias didácticas innovadoras en la enseñanza de las ciencias. Estudio de caso: los maestros del Patronato de Educación Rural de Valencia (1958-1985). Enseñanza de las Ciencias, 35(2), 109-126.

Garmendia, M. y Guisasola, J. (2015). Alfabetización científica en contextos escolares: El Proyecto Zientzia Live! Revista Eureka sobre Enseñanza y Divulgación de las Ciencias, 12(2), 294310.

Gil, D., Carrascosa, J., Furió, C. y Martínez-Torregrosa, J. (1991). La enseñanza de las Ciencias en la Educación Secundaria. Barcelona: Horsori.

Gil-Pérez, D., Macedo, B., Martínez-Torregrosa, J., Sifredo, C., Valdés, P. y Vilches, A. (Eds.) (2005). ¿Cómo promover el interés por la cultura científica? Una propuesta didáctica fundamentada para la educación científica de jóvenes de 15 a 18 años. Santiago de Chile. OREALC/UNESCO.

Gómez J.A. e Insausti M.J. (2004). El ciclo reflexivo cooperativo: un modelo didáctico para la enseñanza de las ciencias. Revista Electrónica de Enseñanza de las Ciencias, 3(2), 148-160.

Hodson, D. (2003). Time for action: science education for an alternative future, International Journal of Science Education, 25(6), 645-670. https://doi.org/10.1080/09500690305021.

Hodson, D. (2011). Looking to the Future. Building a Curriculum for Social Activism. Rotterdam: Sense Publishers

Hugo, D., Adúriz-Bravo, A. y Sanmartí, N. (2013). Estilos de trabajo emocional del futuro profesorado de ciencias durante el prácticum. Enseñanza de las Ciencias, 31(1), 151-168.

Ibáñez, V.E. y Gómez, I. (2005) La interacción y la regulación de los procesos de enseñanzaaprendizaje en la clase de ciencias: análisis de una experiencia. Enseñanza de las Ciencias, 23(1), 97-110.

Kim, M. y Tan, H.T. (2013). A collaborative problem-solving process through environmental field studies. International Journal of Science Education, 35(3), 357-387.

King, D., Ritchie, S., Sandhu, M. y Henderson, S. (2015). Emotionally intense science activities. International Journal of Science Education, 37(12), 1886-1914.

Klemm, J., y Neuhaus, B. J. (2017). The role of involvement and emotional well-being for preschool children's scientific observation competency in biology. International Journal of Science Education, 39(7), 863-876.

León, B., Felipe, E., Iglesias, D. y Latas, C. (2011). El aprendizaje cooperativo en la formación inicial del profesorado de Educación Secundaria. Revista de Educación, 354, 715-729. 
Logan, M.R. y Skamp, K.R. (2013). The impact of teachers and their science teaching on students 'science interest': a four-year study. International Journal of Science Education, 35(17), 2879-2904.

Lu, Y., Chen, H., Hong, Z. y Yore, L. (2016) Students' awareness of science teachers' leadership, attitudes toward science, and positive thinking. International Journal of Science Education, 38(13), 2174-2196.

Marco, B. (2000). La alfabetización científica. En Perales F., Cañal P. (Eds), Didáctica de las Ciencias Experimentales (pp. 141-164). Alcoy: Marfil.

Martínez, M., Esteban, F. y Burraxais, M.R. (2011). Escuela, profesorado y valores. Revista de Educación extraordinario 2011. Educación, valores y democracia, 95-113.

Mata, P. y Ballesteros, B. (2012). Diversidad cultural, eficacia escolar y mejora de la escuela: encuentros y desencuentros. Revista de Educación 358, 17-37.

Mellado, V., Borrachero, B., Brigido, M., Melo, L., Dávila, A., Cañada, F., Conde, C., Costillo, E., Cubero, J., Esteban, R., Martínez, G., Ruiz, C., Sánchez, J., Garritz, A., Mellado, L., Vázquez, B., Jiménez, R. y Bermejo, L. (2014). Las emociones en la enseñanza de las ciencias. Enseñanza de las Ciencias, 32(3), 11-36.

Melo, L., Cañada, F. y Mellado, V. (2017). Exploring the emotions in Pedagogical Content Knowledge about the electric field. International Journal of Science Education, 39(8), 1-20.

Moliner, O., Sales, A., Ferrández, R., Moliner, R. y Roig, R. (2012). Las medidas específicas de atención a la diversidad en la Educación Secundaria Obligatoria (ESO) desde las percepciones de los agentes implicados, Revista de Educación, 358, 197-217.

Navarro, M. Navarro, M., Förster, C., González, C. y González-Pose, P. (2016). Attitudes toward science: measurement and psychometric properties of the Test of Science-Related Attitudes for its use in Spanish-speaking classrooms. International Journal of Science Education, 38(9), 1459-1482.

Perales, F. J. y Cañal P. (2000). Didáctica de las ciencias experimentales. Teoría y práctica de la enseñanza de las ciencias. Alcoy: Marfil.

Perseguer, C. (2012). Identificación de los elementos de gestión del aula mediante la investigaciónacción. Enseñanza de las Ciencias, 30(3), 257-279.

Raved, L. Y Assaraf, O.B.Z. (2011). Attitudes towards science learning among $10^{\text {th }}$-grade students: a qualitative look. International Journal of Science Education, 33(9), 1219-1243.

Retana-Alvarado, D. A., De Las Heras Pérez, M. A., Vázquez-Bernal, B. y Jiménez-Pérez R. (2018). El cambio en las emociones de maestros en formación inicial hacia el clima de aula en una intervención basada en investigación escolar, Revista Eureka sobre Enseñanza y Divulgación de las Ciencias, 15(2), 2602-15.

Rivas, M. (1986). Factores de eficacia escolar: una línea de investigación didáctica. Bordón-Revista de Pedagogía, 264, 693-708.

Rocard, M., Csermely, P., Walwerg-Henriksson, H. y Hemmo, V. (2007). Enseñanza de las ciencias ahora: Una nueva pedagogía para el futuro de Europa, Informe Rocard. Comisión europea, ISBN 978-92.

Rodrigues, A. y Mattos C. (2011). Contexto, negociación y actividad en una clase de física. Enseñanza de las ciencias, 29(2), 263-274.

Rodríguez, D., Armengol, C. y Meneses, J. (2017). La adquisición de las competencias profesionales a través de las prácticas curriculares de la formación inicial de maestros. Revista de Educación, 376, 229-251.

Sammons, P., Hillman, J. Y Mortimore, P. (1995). Key characteristics of effective schools: a review of school effectiveness research. OFSET and University of London, Institute of Education.

Sanz, I., Llorens, J. y Llorens J.A. (2012). La caracterización del ambiente de aprendizaje en un laboratorio de Química General mediante métodos de investigación social. Enseñanza de las Ciencias, 30(1), 5-22. 
Silva, D. y Mcneill, K. (2016). Secondary science students' beliefs about class discussions: a case study comparing and contrasting academic tracks. International Journal of Science Education $38(12), 2047-2068$.

Solbes, J. (2011). ¿Por qué disminuye el alumnado de ciencias? Alambique, 67, 53-61.

Solbes, J. y Vilches, A. (1997). The STS interactions and the teaching of physics and chemistry. Science Education, 81(4), 377-386.

Solbes, J. y Vilches, A. (2004). Papel de las relaciones entre ciencia, tecnología, sociedad y ambiente en la formación ciudadana. Enseñanza de las Ciencias, 22(3), 337-348.

Tiago, D. y Buso, C. (2016). Dinâmicas de grupo em aulas de biología: uma proposta motivacional para a aprendizagem. Revista Electrónica de Enseñanza de las Ciencias, 15(1), 147-166.

Vázquez, A. y Manassero, M.A. (2011). El declive de las actitudes hacia la ciencia de los estudiantes: un indicador inquietante para la educación científica. Revista Eureka, 5(3), 274292.

Vilches, A. (2007). Una unidad clave para la implicación del alumnado: ¿Cómo empezar?, Alambique 52, 28-38.

Vilches, A. y Gil Pérez, D. (2007). La necesaria renovación de la formación del profesorado para una educación científica de calidad, Tecné, Episteme y Didaxis 22, 67-85.

Vilches A. y Gil Pérez, D. (2009). Una situación de emergencia planetaria a la que debemos y podemos hacer frente. Revista de Educación número extraordinario 2009, 101-122. DOI: 10.4438/1988-592X-0034-8082-RE.

Vilches, A. y Gil Pérez, D. (2011). El trabajo cooperativo en las clases de ciencias: una estrategia imprescindible pero aún infrautilizada. Alambique, Didáctica de las Ciencias Experimentales, 69, 73-79.

Wang, C. L. y Liou, P. Y. (2017). Students' motivational beliefs in science learning, school motivational contexts, and science achievement in Taiwan. International Journal of Science Education 39(7), 898-917.

Welch, W. (1985). Research in science education: Review and recommendations, Science Education, 69(3), 421-447.

Wubbels, T. y Brekelmans, M. (2012). Teacher-students relationships in the classroom, Second international handbook of science education, 1241-1255. Netherlands: Springer.

Yung, B.H., Zhu, Y., Ling Wong, S., Wai Cheng, M. y Yin Lo, F. (2013). Teachers' and students' conceptions of good science teaching. International Journal of Science Education, 35(14), $2435-2461$.

\section{CÓMO CITAR ESTE ARTÍCULO}

Espadero, I. y Vilches, A. (2018). Clima del aula en la Educación Científica. Didáctica de las ciencias experimentales y sociales, 35, 59-76. DOI: 10.7203/DCES.35.11410. 